\title{
In Situ ZnO Speciation Changes Towards Zn-Sulfide and Zn- Phosphate within Urban Wastewater Environments
}

\author{
M.A. GomeZ-GONZALEZ ${ }^{1,2 *}$, M.A. KORONFEL ${ }^{1}$, J.E. \\ PARKER $^{2}$, P.D. QUINN ${ }^{2}$, M.L. YALLOP ${ }^{3}$, M.P. RYAN ${ }^{1}$, \\ A.E. PORTER ${ }^{1}$ \\ ${ }^{1}$ Department of Materials, Imperial College London, UK \\ ${ }^{2}$ Diamond Light Source Ltd., Didcot, Oxfordshire, UK \\ (*correspondence: miguel.gomez- \\ gonzalez@diamond.ac.uk) \\ ${ }^{3}$ School of Biological Sciences, Bristol University, UK
}

Zinc oxide engineered nanomaterials ( $\mathrm{ZnO}$ ENMs) are incorporated into a wide range of consumer products, such as sunscreens, personal care products, and anti-bacterial agents, raising concerns about their potential impact as a pollutant following their inevitable release into the environment, through wastewater treatment plants. Understanding the kinetics and extent of $\mathrm{ZnO}$ ENM transformation within sewage waters is then critical to better predicting the impacts of treatment processes, as well as the potential environmental toxicity following subsequent release.

We have applied a cutting-edge spatially-resolved methodology [1], based on in situ X-ray fluorescence microscopy, to observe real-time dissolution, morphological and chemical evolution of $\mathrm{ZnO}$ nanorods within short incubation times (1-3 hours) in influent water and primary sludge collected from an urban sewage plant. Complete transformation of isolated $\mathrm{ZnO}$ hotspots into insoluble $\mathrm{ZnS}$ occurred after only 1 -hour in influent water, suggesting that they become deactivated in this medium; larger aggregates of $\mathrm{ZnO}$ transformed only partially, with small contributions of $\mathrm{Zn}$ bound to phosphate ligands and $\mathrm{Zn}$ adsorbed to $\mathrm{Fe}-$ oxyhydroxide phases around their surfaces. There is evidence that the $\mathrm{ZnO}$ nanorods initially transformed to $\mathrm{ZnS}$ within primary sludge at 1 -hour and evolved towards a more complex structure with a $\mathrm{Zn}_{3}\left(\mathrm{PO}_{4}\right)_{2}$ shell surrounding a $\mathrm{ZnO}$ core after 3-hours. This work highlights the need for timeresolved studies of ENMs transformations in environmental media over longer time scales, evaluating the impact of these transformed species to microorganisms. Further research is needed to determine the impacts of these modified nanomaterials on selected biota at a point of exposure, rather than the traditional approach using pristine nanomaterials.

[1] Gomez-Gonzalez, M. A. et al.; ACS Nano 2019, 13 (10), 11049-11061; doi: 10.1021/acsnano.9b02866 